\title{
GALILEAN TRANSFORMATION EXPRESSED BY THE DUAL FOUR-COMPONENT NUMBERS
}

\author{
V. MAJERNíK \\ Institute of Mathematics, Slovak Academy of Sciences \\ 81478 Bratislava, Śtefánikova 49, Slovak Republic \\ and \\ Department of Theoretical Physics, Palacký University \\ Tr̃. Svobody 26, 77461 Olomouc, Czech Republic
}

(Received Augusl 19, 1994; revised version January 26, 1995)

\begin{abstract}
We express the special Galilean transformation in the algebraic ring of the dual four-component numbers.
\end{abstract}

PACS numbers: 02.10.Lh, 02.10.Nj

\section{Introduction}

In the previous paper [1] we have presented the relations between different two-component number systems and the corresponding two-dimensional space-time symmetry groups. In order to find similar relations between the physical four-dimensional space-time symmetry groups and the corresponding number systems we need to extend the existing two-component number systems to the four-component ones. As is well known the Lorentz transformation of a boost of $v$ in the $x$ direction can be written as a relation between the four-component numbers called quaternions [2]

$$
x^{\prime}=\exp \left[-\left(i e_{1} \phi\right) / 2\right] x \exp \left[-\left(i e_{1} \phi\right) / 2\right], \quad \tanh \phi=v / c,
$$

where $x=c t+i x_{j} e_{j}$ and $e_{1}, e_{2}, e_{3}$ are the quaternion units. Here the question arises whether it does exist such a four-component number by which one can express the special Galilean transformation in a similar form as (1). We will show that this can be achieved by means of the dual four-component number system which represents the extension of the dual two-component number system. Ilowever, before doing this let us recall some facts regarding the two-component number systems [1].

It is well known that a two-component number system forming an algebraic ring can be written in the form $Z=a+\varepsilon b, a, b \in R$, where $\varepsilon$ is a certain "imaginary" unit. The algebraic structure of a ring demands that the product of each two-component numbers $Z_{1}=a+\varepsilon b$ and $Z_{2}=c+\varepsilon d$,

$$
Z_{1} Z_{2}=(a+\varepsilon b)(c+\varepsilon d)=a c+\varepsilon(a d+b c)+\varepsilon^{2} b d,
$$


belongs to the ring as well. Therefore, $\varepsilon^{2}=\beta+\varepsilon \gamma, \beta, \gamma \in R$. An important mathematical theorem states that any possible two-component number can be reduced to one of the following three types [3]. The "imaginary" unit will be denoted by $i, \lambda$ or $\mu$, respectively, in each of the three specific cases listed below:

(i) the complex numbers $Z_{\mathrm{c}}=a+\varepsilon b$ with $\varepsilon^{2}=i^{2}=-1$,

(ii) the binary numbers [3] (called also the double [4] and anomal-complex [5]) $Z_{\mathrm{b}}=a+\varepsilon b ;$ with $\varepsilon^{2}=\lambda^{2}=1$ and

(iii) the dual numbers, $Z_{\mathrm{d}}=a+\varepsilon b$, with $\varepsilon^{2}=\mu^{2}=0$.

The criterion for the membership to one of these two-component number systems is given by the sign of the expression $Q=(\beta+\gamma / 4)$. If $Q$ is negative, positive or zero then we get the complex, binary or dual numbers, respectively.

The usual operations done for the complex numbers, such as absolute value, goniometric form, and Euler formula, have corresponding operations in other two-component systems. For a number, $Z=a+\varepsilon b$, from any of these three number systems its conjugate is given as $Z^{*}=a-\varepsilon b$ and its absolute value is defined as $|Z|=\sqrt{\left|Z Z^{*}\right|}$. All $Z$ can be expressed also in the goniometric form $Z=|Z| \exp (\varepsilon \phi), \phi=F(a / b)$, where $\phi=\arctan (a / b)$ holds for the complex, $\phi=\operatorname{arctanh}(a / b)$ for the binary and $\phi=(a / b)$ for the dual numbers, respectively.

\section{Four-component number systems and Galilean transformation}

The four-dimensional extension of the complex two-component number system represents the system of Hamiltonian quaternions. A Hamiltonian quaternion is defined as [2]

$$
q=q_{0}+q_{1} e_{1}+q_{2} e_{2}+q_{3} e_{3}
$$

with the multiplication scheme for the quaternion units

$$
\boldsymbol{e}_{i} \boldsymbol{e}_{j}=-\delta_{i j}+\varepsilon_{i j k} \boldsymbol{e}_{k}
$$

where $\varepsilon_{i j k}$ represent the components of totally antisymmetric tensor. IIamiltonian quaternions have a large field of application in mathematical physics (see, e.g. [2]). The corresponding four-component numbers which represent the four-dimensional extension of the two-component binary numbers are the hyperbolic quaternions. They have a wide use especially in supersonic fluid mechanics [6]. Another form of quaternions, called the biquaternions, play an important role by the compact and elegant formulation of special relativity, electrodynamics and supersymmetric theories (see, e.g. [7]).

Next we will show that Galilean transformation can also be written in the frame of the four-dimensional number system which represents a four-dimensional extension of the two-component dual numbers. The element of this system is a dual four-component number (DFN for short) defined as

$$
Q_{d}=q_{0}+q_{1} i+q_{2} j+q_{3} k
$$

with the multiplication scheme for the dual units $i, j, k$

$$
i j=j i=i k=k i=j k=k j=0, \quad i i=j j=k k=0 .
$$


The product of two DFNs $Q$ and $\boldsymbol{Q}^{\prime}$ is again a DFN

$$
\boldsymbol{Q}^{\prime \prime}=Q Q^{\prime}=q_{0} q_{0}^{\prime}+\left(q_{0} q_{1}^{\prime}+q_{1} q_{0}^{\prime}\right) i+\left(q_{0} q_{2}^{\prime}+q_{2} q_{0}^{\prime}\right) j+\left(q_{0} q_{3}^{\prime}+q_{3} q_{0}^{\prime}\right) k,
$$

so that the set of DFNs forms an algebraic ring. The ratio $\boldsymbol{R}$ of two DFNs form

$\boldsymbol{Q}=q_{0}+q_{1} i+q_{2} j+q_{3} k$ and $\boldsymbol{Q}^{\prime}=q_{0}^{\prime}+q_{1}^{\prime} i=q_{2}^{\prime} j+q_{3}^{\prime} k$ can be written in the

$$
R=Q^{\prime} / Q=Q^{\prime} Q^{*} / Q Q^{*}=Q^{\prime} Q^{*} / q_{0}^{2}
$$

where the conjugate DFN is defined as

$$
Q^{*}=q_{0}-q_{1} i-q_{2} j-q_{3} k
$$

and the norm of $Q$, given as $Q Q^{*}$, is $q_{0}^{2}$.

DFNs represent the natural number system for the expression of special Galilean transformation

$$
r^{\prime}=v t, \quad r^{\prime}=\left(x^{\prime}, y^{\prime}, z^{\prime}\right) ; \quad r=(x, y, z) ; \quad v=\left(v_{1}, v_{2}, v_{3}\right),
$$

where $r^{\prime}$ and $r$ are the position vectors in the inertial systems moving with velocity $v$ relative to each other and $t$ is time. If we express the space-time in the form of the DFN

$$
\boldsymbol{X}=c t+x i+y j+z k,
$$

where $i, j, k$ obey the multiplication rules of the dual units (2) then the special Galilean transformation (3) can be written in the following form

$$
\boldsymbol{X}^{\prime}=\exp \left(-\Phi_{1}-j \Phi_{2}-k \Phi_{3}\right)(c t+x \boldsymbol{i}+y \boldsymbol{j}+z \boldsymbol{k}),
$$

where $\boldsymbol{X}^{\prime}=c t^{\prime}+x^{\prime} j+z^{\prime} k$ and $\Phi_{1}=v_{1} / c, \Phi_{2}=v_{2} / c$ and $\Phi_{3}=v_{3} / c$. Taking into account that $i^{n}=j^{n}=k^{n}=0$ for $n>1$, we get

$$
\exp \left(-i \Phi_{1}-j \Phi_{2}-k \Phi_{3}\right)=1-i \Phi_{1}-j \Phi_{2}-k \Phi_{3} .
$$

Equation (4) can be written in the familiar form of Galilean transformation (3),

$$
x^{\prime}=x-v_{1} t, \quad y^{\prime}=y-v_{2} t, \quad z^{\prime}=z-v_{3} t
$$

and $t^{\prime}=t$, which leaves the expression $\boldsymbol{X} \boldsymbol{X}^{*}=(c t)^{2}$ unchanged. This is in agreement with the assumption of the Newtonian physics requiring the existence of the absolute time. Equation (4) can be rewritten also in the form

$$
\boldsymbol{X}^{\prime}=G(\Phi) \boldsymbol{X},
$$

where $G(\Phi)=\exp (-i \Phi)$ stands for the operator of Galilean transformation. The successive application of two Galilean transformations described by the operators $G\left(\Phi_{2}\right)$ and $G\left(\Phi_{1}\right)$,

$$
\boldsymbol{X}^{\prime}=G\left(\Phi_{1}\right) \boldsymbol{X} \quad \boldsymbol{X}^{\prime \prime}=G\left(\Phi_{2}\right) \boldsymbol{X}^{\prime}
$$

is equivalent to an application of a single operator

$$
\boldsymbol{X}^{\prime \prime}=G\left(\Phi_{3}\right) \boldsymbol{X},
$$

where $G\left(\Phi_{3}\right)=G\left(\Phi_{1}\right) G\left(\Phi_{2}\right)=G\left(\Phi_{2}\right) G\left(\Phi_{1}\right)=G\left(\Phi_{1}+\Phi_{2}\right)$ which implies the addition theorem for the velocities in the Galilean transformation. Equation (4) resembles the formula for the rotation of a complex vector, $Z=x+i y, Z^{\prime}=R(\phi) Z$, where $R(\phi)=\exp (i \phi)$ represents the operator of rotation and $\phi$ is the angle of rotation. As is well known the set of the operators $R(\phi)$ forms a group. Similarly, 
the set of the Galilean operators $G(\Phi)$ forms a group with the continuous parameter $\Phi=v / c$ the "angle" of the rotation of the dual space-time $\boldsymbol{X}$.

The formulation of Galilean transformation by means of DFNs has not only a certain elegance and aesthetic appeal but it shows that the linkage between the space and time exists also in the Newtonian physics. Moreover, it may have a considerable heuristic value for the study of the underlying mathematical formalism of physical laws.

\section{A possible use of two-component numbers in integration}

Let us finish with a mathematical curiosity connected with the dual two-component numbers. As is well known the Euler and Moivre formulas for the complex, binary and dual numbers are

$$
\begin{array}{ll}
\exp (i \phi)=\cos \phi+i \sin \phi, & \exp (i \phi)^{n}=(\cos \phi+i \sin \phi)^{n}, \\
\exp (\lambda \phi)=\cosh \phi+\lambda \sinh \phi, & \exp (\lambda \phi)^{n}=(\cosh \phi+\lambda \sinh \phi)^{n}, \\
\exp (\mu \phi)=1+\mu \phi, & \exp (\mu \phi)^{n}=1+n \mu \phi,
\end{array}
$$

respectively. By means of Eqs. (5a), (5b) we get the identities $\phi=x$

$$
\begin{aligned}
& \sin x=[\exp (i x)-\exp (-i x)] / 2 i, \sinh x=[\exp (\lambda x)-\exp (-\lambda x)] / 2 \lambda, \\
& \cos x=[\exp (i x)+\exp (-i x)] / 2, \cosh x=[\exp (\lambda x)-\exp (-\lambda x)] / 2 .
\end{aligned}
$$

Ilowever, Eq. (5c) makes possible to express also the variable $x$ and the real number $1 \mathrm{in}$ a form containing only the combinations of exponential functions

$$
x=[\exp (\mu x)-\exp (-\mu x)] /(2 \mu), \quad 1=[\exp (\mu x)+\exp (-\mu x)] / 2 .
$$

Therefore, a special function of the following type

$$
F(x)=P_{1}(x) P_{2}[\exp (x)],
$$

where $P_{1}$ and $P_{2}$ stand for polynomials, can be expressed as a sum of exponential functions

$$
F(x)=E_{1}(x)+E_{2}(x)+\ldots+E_{n}(x),
$$

the general form of which is

$$
E_{i}=[\exp (a+b \mu)] /(c \mu), \quad i=1,2, \ldots n, \quad a, b, c \in R .
$$

The decomposition (5e) makes possible to perform the integration of the functions $F(x)$ simply as a sum of exponential functions. As an elementary example let us determine the integral

$$
I=\int \mathrm{d} x x \exp (x)
$$

Using Eq. (5d) we obtain 


$$
I(x)=\int \mathrm{d} x \exp [(1+\mu) x] /(2 \mu)+\int \mathrm{d} x \exp [(1-\dot{\mu}) x] /(2 \mu) .
$$

Taking into account that $(1+\mu)(1-\mu)=1$ and using again Eq. (5d) we get finally

$$
\begin{aligned}
& I(x)=\exp (x)[(1-\mu) \exp (\mu x) /(2 \mu)-(1+\mu) \exp (-\mu x) /(2 \mu)]= \\
& \quad \exp (x)\{[\exp (\mu x)-\exp (-\mu x)] /(2 \mu)-[\exp (\mu x)+\exp (-\mu x)] /(2 \mu)\}= \\
& (x-1) \exp (x) .
\end{aligned}
$$

We realize that here the integration by means of dual numbers requires larger procedure than the use of other common integration-methods. Nevertheless, it shows an interesting and curious way of integration of a certain type of function in the form of a sum of simple exponential functions. We note, that using the all two-component number systems, a considerable large class of functions of the type

$$
F(x)=P_{1}(x) P_{2}(\sin x, \cos x) P_{3}(\sinh x, \cosh x) P_{4}(\exp (x)),
$$

where $P_{1}, P_{2}, P_{3}$ and $P_{4}$ stand for polynomials, can be written as a sum of certain exponential functions. However, a multicomponent number system, whose detailed mathematical analysis would exceed the scope of this paper, will be subject of a subsequent paper.

\section{References}

[1] V. Majernik, Acta Phys. Pol. A 86, 291 (1994).

[2] J.L. Synge, Quaternions, Lorentz Transformation and Conway-Dirac-Eddington Malrices, Dublin Institute for Advanced Studies, Dublin 1972.

[3] I.L. Kantor, V.N. Solodownikov, Hypercomplexe Zahlen, Teubner, Leipzig 1978.

[4] W. Benz, Vorlesungen über Geometrie der Algebren, Springer, Berlin 1973.

[5] I.M. Yaglom, Complex Numbers in Geometry, Academic Press, New York 1968.

[6] M. Laurentiev, B. Chabat, Effects Hydrodynamiques et Modéles Mathematiques, Mir, Moscow 1980.

[7] R. Anderson, G. Joshi, Physics Essays 6, 308 (1993). 